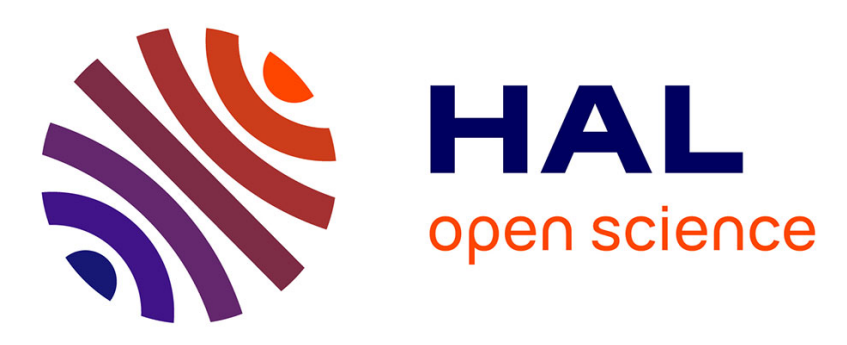

\title{
Dynamic Round-Trip Engineering in the context of FOMDD
}

\author{
Glenn Cavarlé, Alain Plantec, Steven Costiou, Vincent Ribaud
}

\section{To cite this version:}

Glenn Cavarlé, Alain Plantec, Steven Costiou, Vincent Ribaud. Dynamic Round-Trip Engineering in the context of FOMDD. 11th International Workshop on Smalltalk Technologies, Aug 2016, Prague, Czech Republic. pp.1 - 7, 10.1145/2991041.2991056 . hal-01448784

\section{HAL Id: hal-01448784 \\ https://hal.univ-brest.fr/hal-01448784}

Submitted on 29 Jan 2017

HAL is a multi-disciplinary open access archive for the deposit and dissemination of scientific research documents, whether they are published or not. The documents may come from teaching and research institutions in France or abroad, or from public or private research centers.
L'archive ouverte pluridisciplinaire HAL, est destinée au dépôt et à la diffusion de documents scientifiques de niveau recherche, publiés ou non, émanant des établissements d'enseignement et de recherche français ou étrangers, des laboratoires publics ou privés. 


\title{
Dynamic Round-Trip Engineering in the context of FOMDD
}

\author{
Glenn Cavarlé Alain Plantec Steven Costiou Vincent Ribaud \\ Univ. Bretagne-Occidentale, UMR CNRS 6285, Lab-STICC, F-29200 Brest, France \\ \{glenn.cavarle, alain.plantec, steven.costiou, vincent.ribaud\}@univ-brest.fr
}

\begin{abstract}
In the context of Feature-Oriented Model-Driven Development, round-trip engineering remains challenging because of the one-to-many relationship that exists between a source model and its implementation parts. In this paper, we present CrossFabrik, an approach that allows round-trip engineering with dynamic assessment of generated implementations. Such an approach relies on the reflective capability of the development environment. An implementation of our approach within Pharo is also presented.
\end{abstract}

Keywords feature-oriented development, round-trip engineering, model driven engineering, smalltalk

\section{Introduction}

Model-Driven Development (MDD) promotes models as first-class entities in software design. Feature-Oriented Development (FOD) is a paradigm used in Software Product Line (SPL) that operates through features composition to produce software variants. Such a paradigm imposes a one-to-many relationship between a model and the executable artefacts which are produced from it. FeatureOriented Model-Driven Development (FOMDD) [1, 2] is a blend of FOD and MDD that exploits features composition to create models and that use model transformations to produce software variants.

Basically, MDD, FOD and FOMDD share the same protocol to assess a software during development. The source artefacts cannot be directly executed. Instead, transformations have to be performed to produce an executable artefact from the source artefacts. Hence, from the editing of source artefacts to the execution of the generated ones, a typical development cycle can be described as follows. First, the source code has to be generated from the source artefacts, then the source code has to be compiled and the resulting executable artefact has to be deployed before it can be actually run. In case of an issue occurring during the execution, the faulty running context has to be observed to discover the defective source artefact. We characterize this step as the feedback process. After the developer has fixed the source artefact, the assessment protocol has to be achieved again.

The consequence of such a process is a break between the editing of the source artefact and the execution of the gener- ated artefact. After the developer has fixed the source artefact, it may be tedious to come back to the original execution context and to continue a validation process. Moreover, the developer faces a traceability issue between a malfunction that is observed during execution and the source artefact that should be fixed [3].

Round-Trip Engineering can be used to allow the editing of generated artefacts while keeping source artefacts synchronized. The goal of round-trip engineering is keeping a number of artefacts, such as models and code, consistent by propagating changes among the artefacts. Round-trip engineering is a special case of synchronization that can propagate changes in multiple directions, such as from models to code and vice versa. Round-trip engineering is hard to achieve in a general setting due to the complexity of the non-isomorphic mappings between the artefacts [4], especially in the context of FOMDD.

This paper presents a dynamic Round-Trip Engineering in the context of FOMDD. This solution is part of CrossFabrik, our engineering environment for cross-platform software design. The goal is to reduce the development cost by maximizing the use of the development environment before the platform specific artefacts are generated. With CrossFabrik the model editing as well as the simulation of the software variants are performed closely within the development environment.

The three contributions of this paper are:

- We present the CrossFabrik language, our specific language dedicated to model specification in the context of FOMDD.

- We describe our dynamic Round-Trip Engineering which preserves the one-to-many relationship between a source model and the executable artefacts.

The remainder of this paper is organized as follows: the next section recalls the main concepts regarding feature modelling. Section 3 introduces the CrossFabrik language that is used to specify source models. Section 4 presents a motivating example that illustrates a typical situation that arises in FOMDD and that highlights specific issues in software assessment. In Section 5, we describe our approach regarding software assessment in the context of FOMDD. A focus on the Pharo reflective model is given in Section 6. Section 7 
explains how the dynamic Round-Trip Engineering is implemented using the Pharo reflective capabilities. Then, we present in Section 8 the dedicated tools to edit models and how we reuse the Pharo infrastructure to manipulate and debug executable artefacts. Finally, in Section 9, related work is presented before concluding in Section 10.

\section{Background: Feature Modelling Overview}

The concept of feature modelling comes from Software Product Line [5].

A feature has been defined as "a logical unit of behaviour specified by a set of functional and non-functional requirements" [8] and usually referred as a user-visible aspect of a software.

A Feature Model $[6,7]$ is a logical representation of a software family. A software family consists in a set of software which share mandatory and optional features. A Feature Model is specified as a tree of named nodes.

From a Feature Model, multiple software variants can be defined. A software variant is itself defined by a configuration. A configuration is a feature selection among the nodes of the Feature Model.

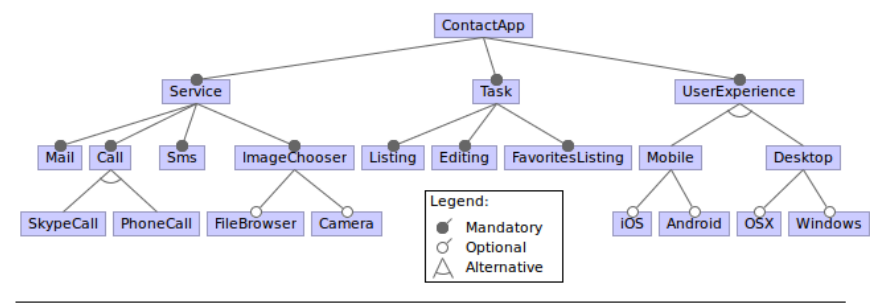

Figure 1. An example of a Feature Model.

Figure 1 shows the Feature Model of the ContactApp software family. Each node specifies a feature. In this example, a particular configuration could be made of the following features: Mail, Phone Call, Camera, Task and Android. Another configuration could be define to use the $i O S$ feature instead of the Android one.

Implementing features is under the responsibility of the development environment. Each environment provides its own approach to implement software artefacts according to specified features.

The following section presents the meta-model that is used in CrossFabrik to specify the software artefacts.

\section{Implementing Features With CrossFabrik}

The CrossFabrik language consists in a meta-model that integrates the concept of feature. It allows the implementation of a Feature Model. The goal is to specify the mapping between features and supporting software artefacts.
The main elements of the meta-model are depicted in Figure 2. Regarding the structural aspects, the CrossFabrik language is EMOF-inspired [9]. The FabrikElement is the root abstraction for elements such as a class, an attribute and a method description. These elements are represented in CrossFabrik by, respectively FabrikClass, FabrikAttribute and FabrikMethod.

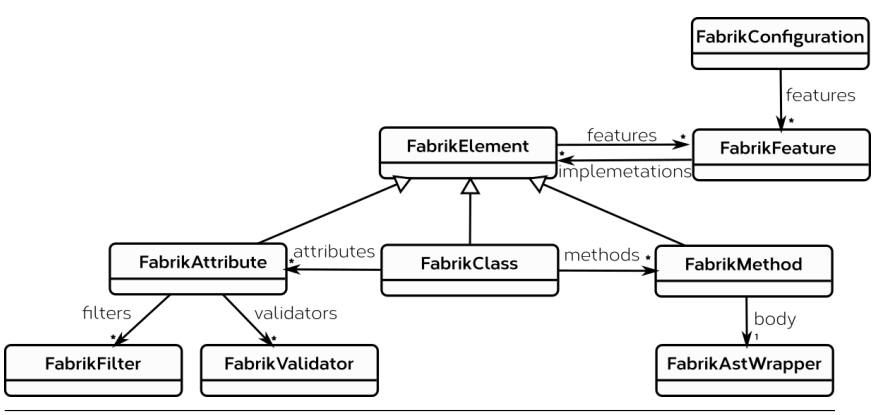

Figure 2. CrossFabrik meta-model fundamentals

The FabrikAttribute element describes an instance variable. Such an element can be associated with data validators and with data filters to specify the way an attribute value can be accessed.

The business behaviour is described through the FabrikMethod element. A FabrikAstWrapper is associated with a FabrikMethod element through its body property to represent the source code.

A FabrikFeature entity is a reference to a feature which is expressed in a Feature Model. Any element can be associated with multiple features and any feature can be associated with multiple elements.

A particular configuration namely an instance of FabrikConfiguration is made of a set of FabrikFeature instances.

Thus, the production of a software variant consists in three main steps. First, features are specified by a Feature Model. The configurations are defined by selecting relevant features. Then, features can be implemented using elements of the CrossFabrik meta-model. Finally, each software variant can be generated from a CrossFabrik model associated with a particular configuration.

\section{A Motivating Example}

This section presents an example based on a User entity. For all software variants of the software family, a User is made of an email attribute. But, in our example, the developer needs to implement two different variants. The first with a Location feature and the second without it.

Figure 3 depicts a Feature Model for this example. The root feature is named Root. Location is an optional feature which adds the possibility for a user to be geolocalized. In this case, two additional properties are required for the management 
of a User, namely the location field and the updateLocation method.

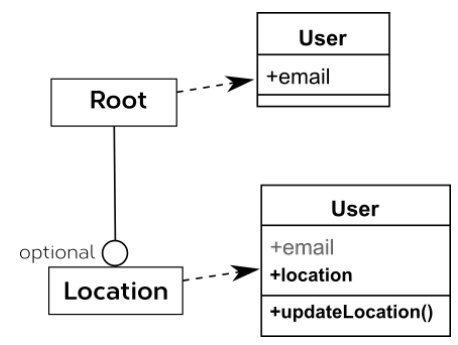

Figure 3. The User model and the associated features.

Given that from Root, there are two possible configurations, as a consequence, there are two possible implementations for one User model. It is depicted by the implementation-of relationship in Figure 4.

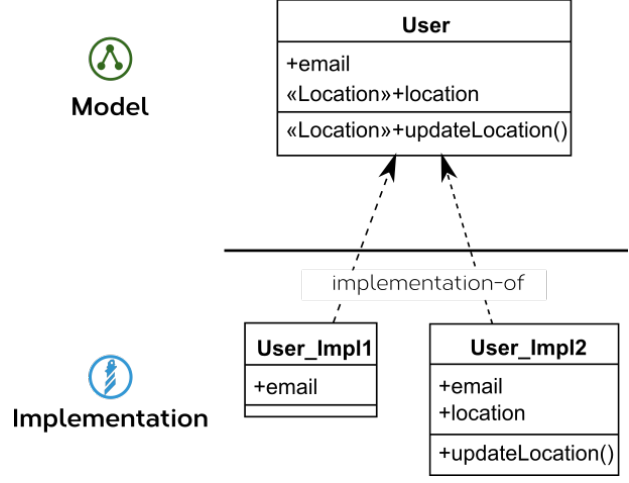

Figure 4. The User model and its two implementations.

These two implementations are automatically generated from the source model together with the specification of the configuration (e.g the actual selection of features).

In currently available Feature-Oriented development environments, after code generation, the generated classes User_Impl1 and User_Impl2 are self-contained and are no longer linked with the related User model.

Typically, this situation implies the following difficulties:

- During the assessment of a particular implementation, each time an issue occurs, the current execution has to be stopped. Then, the User model has to be fixed according to the relevant feature and finally the source code have to be regenerated. This clearly breaks the development flow.

- While debugging the Location feature, a developer may need to dynamically manipulate the User model. But because of the code generation process, this may be impossible.

- A developer may choose to investigate a particular solution at implementation side. As an example, the developer may want to tune the Location feature implementation. But in this case, after the investigation is over, the User model has to be manually synchronized with the new implementation. Moreover, changing the implementation of the base User model (without the Location feature) imposes the validation of all the dependent configurations. If the developer decides to directly change its implementation, then he has to manually maintain the User model and to regenerate the other configurations.

The CrossFabrik approach supports dynamic Model-Driven RTE. It consists in using the same environment at design time as well as at running time. The CrossFabrik approach is presented in the next section.

\section{The CrossFabrik RTE Approach}

With CrossFabrik, the modelling and the execution take place within a unique environment at design time as well as at running time. As depicted by Figure 5, the dynamic RTE consists in an incremental and continuous synchronisation between a source model and intermediate implementation classes used for simulation purpose.

A source model is specified using the CrossFabrik language and implementation classes are generated automatically for each configuration.

In CrossFabrik, information duplication is avoided to allow the dynamic synchronization. A generated implementation class is associated with the model element that describes it. At runtime, this association allows the dynamic synchronization of the source model from any instance of the generated class. As an example, a generated class method does not contain any source code. The method source code is only described in the source model and it is dynamically used during the assessment of the generated class.

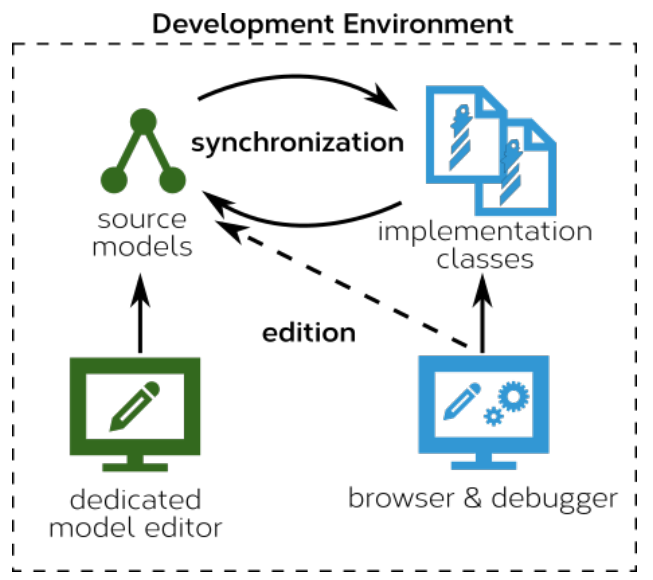

Figure 5. The CrossFabrik RTE approach.

The development cycle can be decomposed in two phases. First, the developer starts to model a software family by using dedicated editors. Depending on the current synchronization strategy in use, the implementation classes can be 
either generated on demand or automatically during the editing. Then, the developer can instantiate the generated implementation classes to assess a software variant.

The CrossFabrik approach deeply relies on reflective capabilities of the development environment especially for the dynamic RTE and for the debugging. For the validation of our approach we use the Pharo Environment.

\section{A Focus On The Pharo Reflectivity}

In Pharo [10], a class, an instance variable, a class variable and a method namely Class, Slot, ClassVariable and CompiledMethod, are first-class objects. Using the reflective capabilities of Pharo, it is possible to dynamically change the structural aspects of a Class. Such a change affects immediately the running system (e.g. all instances of the updated class).

In the scope of a class, Slot and Class Variable instances [11] define the reading and the writing behaviour of instance variables and of class variables. Slot and Class Variable can be subclassed to provide a specific behaviour.

Any object can play the role of a CompiledMethod as long as it is able to responds to the specific message run:with:in: An object that responds to run:with:in: is called a method wrapper or a method proxy [12].

To maintain the dynamic synchronization between CrossFabrik elements and generated implementation classes, the CrossFabrik implementation of RTE relies on slots and method proxies. This RTE implementation is presented in the next section.

\section{The RTE Implementation}

A model may have several implementation variants according to the features set. Then, for one model, the round-trip implementation has to keep multiple implementation classes synchronized. Any RTE implementation automatically manages forward and reverse engineering:

- First, the forward engineering capability. Any change in a model have to be automatically applied to related Pharo classes.

- Second, the reverse engineering capability. Any change in a Pharo class have to be automatically reflected in the source model.

Because of the one-to-many relationship, implementing the reverse engineering can be tedious. A change in a Pharo class can also impact implementation classes generated from other configurations. Moreover, reverse changes have to be controlled and validated before being applied to models. Any ambiguity must be resolved and improper changes must be rejected in order to preserve the model integrity.
The forward and the reverse engineering are implemented by a mediator class named ChangeManager. The implemented mechanisms are depicted in Figure 6:

- Forward engineering involves generating Pharo classes from a source model. This mechanism is event-based. Events are emitted each time a source model is updated. Then, the ChangeManager reacts by propagating changes in the related Pharo classes. The ChangeManager takes care of the one-to-many relationship between the source model and the implementation classes.

- Reverse Engineering is change-based. It relies on the redefinition of the default update behaviour of the generated classes at class definition level as well as at method definition level. In case of a structural change of a class or in case of a method definition change, the default behaviour is prevented, instead, the ChangeManager is asked to update the related source model.

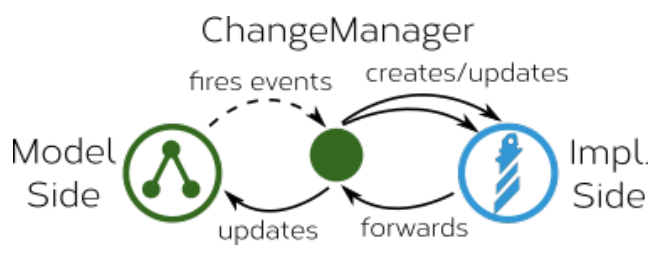

Figure 6. The ChangeManager acts as a mediator object.

\subsection{The Forward Engineering}

The forward engineering takes place each time a source model is updated. When an CorssFabrik element is changed, an event is emitted by the element. The ChangeManager reacts to these events by updating the generated classes.

In order to implement the RTE, CrossFabrik has to manage the way a class is installed in the system and has to change the default behaviour. For that purpose, CrossFabrik implements its own meta-entities to be used in place of Slot, ClassVariable and CompiledMethod to redefine the reading and the writing the behaviour of slots and the way a method is executed.

The use of these CrossFabrik meta-entities for our User example is depicted by Figure 7. Instead of using a Slot, the mail attribute is implemented through an instance of FabrikAttributeSlot. Instead of using a CompiledMethod, the updateLocation method is implemented though an instance of FabrikMethodProxy. Instead of using a ClassVariable, the User source model is referenced by a FabrikClassVariable instance. FabrikAttributeSlot, FabrikClassVariable and FabrikMethodProxy hold a reference to the CrossFabrik elements. 


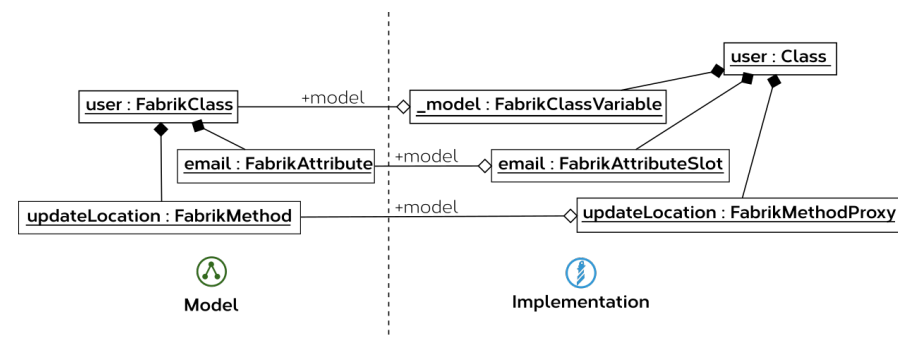

Figure 7. User model instance (left) with related metaentities (right).

The running of a software variant relies on a proxy mechanism from the generated class to the source model. Figure 8 depicts the implementation of the run:with:in: method of the FabrikMethodProxy. It shows that the execution relies only on the source code specified in the source model. First, the method proxy retrieves the AST method node from its model. Then, the AST method node is converted to a CompiledMethod before being executed.

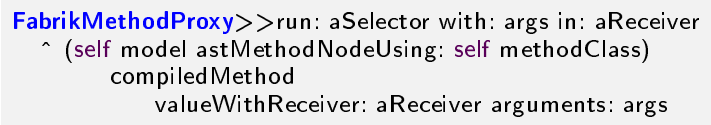

Figure 8. Implementation of the run:with:in: method of the FabrikMethodProxy.

\subsection{The Reverse Engineering}

To perform the reverse engineering, the ChangeManager has to be aware of changes applied in implementation classes. In Pharo, object update is the responsibility of the objects themselves. Thus, we can implement a new update strategy directly in implementation classes.

Two kind of changes have to be intercepted and propagated at model side:

- The structural changes: the addition/deletion of instance variables or of methods of an object.

- The behavioural changes: the modification of a method's body.

In Pharo, all structural changes are delegated to the PharoClassInstaller. In CrossFabrik, the PharoClassInstaller is not directly used by generated classes. Instead, the ChangeManager act as a mediator between the generated classes and the PharoClassInstaller.

When an instance variable is added, the ChangeManager is asked to update the related source model. It first check the validity of the change, then it applies the change on the related source model and finally the forward engineering takes place.

In Pharo, the methods management is under the responsibility of the class itself. When a method is compiled, the class method named addAndClassifySelector:withMethod: is invoked to perform the actual changes. In CrossFabrik, this method is redefined to delegate method changes to the ChangeManager. Figure 9 depicts how the class method $a d$ dAndClassifySelector:withMethod: is redefined. In case of the update of an existing method, the ast of the source model is directly updated. In case of a new method or of a method removing, the request is forwarded to the ChangeManager in order to perform relevant changes on source model.

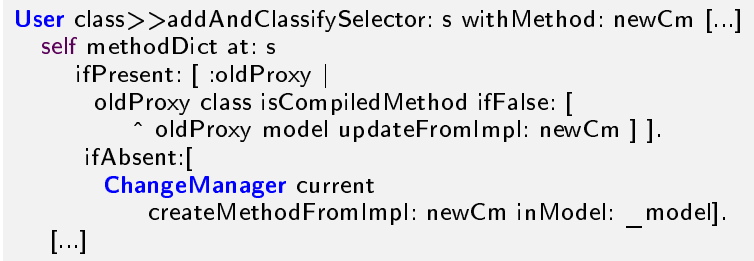

Figure 9. The redefinition of the addAndClassifySelector:withMethod: method.

\section{Tools Support}

The Pharo infrastructure is fully usable with CrossFabrik. However, to ease the edition of source models and the debugging of generated classes, a set a tools has been specifically implemented. These tools are presented in this section.

\subsection{Editing Models}

Dedicated tools including editors and a specific declarative language were implemented to manipulate models. CrossFabrik provides an Integrated Development Environment (IDE) for that purpose.

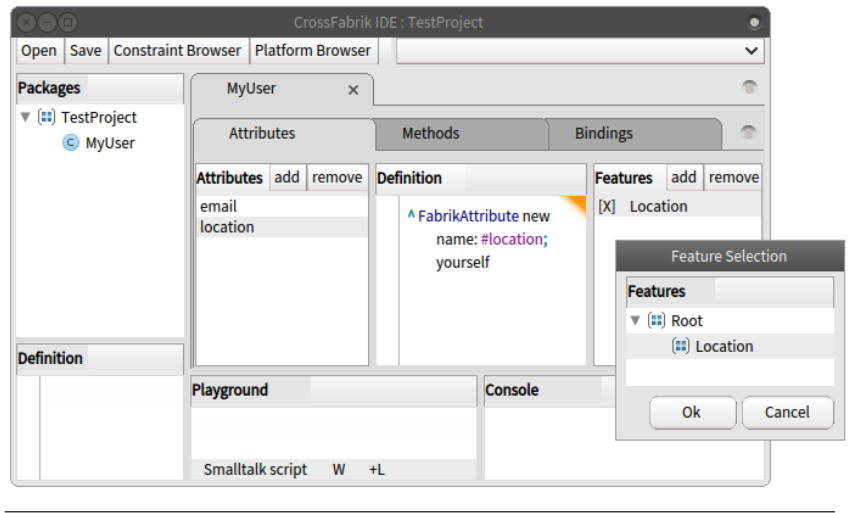

Figure 10. The CrossFabrik IDE.

Figure 10 shows the CrossFabrik IDE for models editing. It is mainly composed at left by a package browser from which models can be opened. Tabulations are presented at the center. They show the content of the models. Attributes and methods can be edited and can be associated with features. The popup window at bottom right shows a view of the Feature Model currently in use. 
From this package browser, the developer can also open a model in a textual mode. Instead of the default model editor, a text editor is opened that contains the textual representation of the selected model.

As an example, the Figure 11 shows the declarative syntax used to describe the User model.

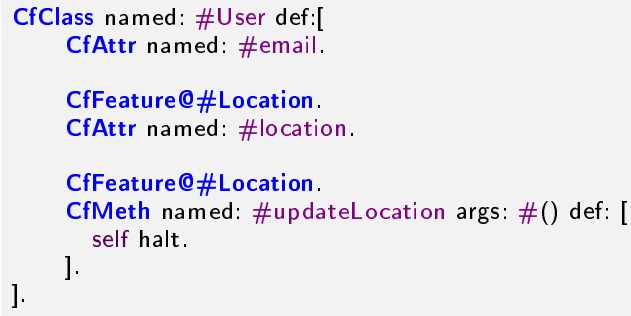

Figure 11. The declarative syntax use to describe the User model.

This declarative syntax can be directly executed within any Pharo editor.

\subsection{Extending The Pharo Debugger}

Pharo provides the GTDebugger [13] that offers a way for developers to adapt visualization to new domains and scenarios. It is object-centric and retrieves the relevant visualizations directly from the inspected object. Thus, the debugger is dynamically adapted when a particular inspected object contains methods that describe new visualization to use.

As an example, Figure 12 shows the debugging of one User implementation.

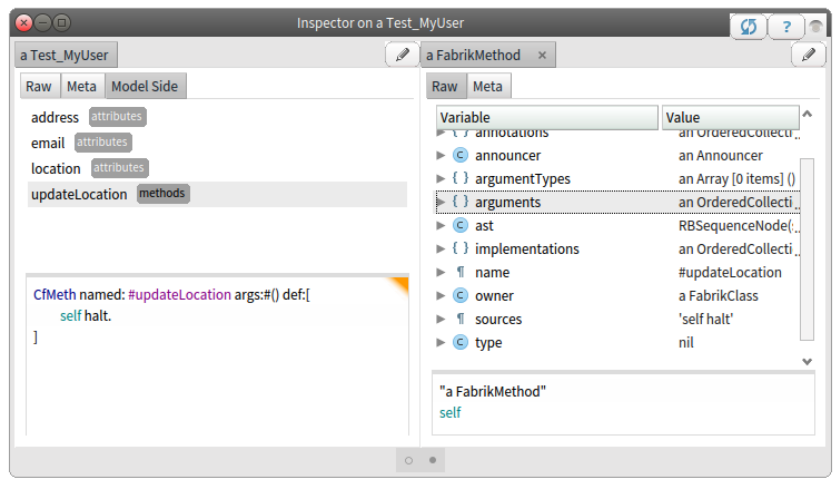

Figure 12. The debugging of one User implementation.

The CrossFabrik debugging visualization is composed of two columns. The first column lists the attributes and the methods of the current inspected object. When an item is selected in the list, its source model is displayed below the list using the CrossFabrik textual representation. This representation can be edited within the browser. The second column displays the structure of the source model. As any other inspected object, the source model can be manipulated and edited.

\section{Related Work}

In this Section we discuss related work on round-trip engineering. As far as we know, CrossFabrik is the only environment that provide dynamic debugging and RTE support in the context of FOMDD.

In [19], a Change-Oriented Advanced Round-Trip Engineering (COARTE) is envisioned. In COARTE, changes are considered as first-class objects and the entire set of changes objects represents the complete history of a software system. As in COARTE, CrossFabrik uses a change-oriented RTE. Our implementation relies on an event model and on the change mechanism provided by Pharo. But we do not maintains the history of changes made. Changes cannot be stored and replayed.

COARTE also provides multiple views on a software system with the distinction between static and runtime views and between design and implementation views. In CrossFabrik this distinction is also made. The CrossFabrik IDE depicts how a software is designed whereas the Pharo code browser and the debugger depict respectively how a software is statically implemented and how implementation instances live in the system.

But COARTE does not address the issue of the synchronization of multiple implementations from the same model. In CrossFabrik, the first requirement regarding the RTE is to maintain this one-to-many relationship. As far as we know, COARTE does not allow such a kind of synchronization.

SelfSync [18] is an approach and tools for providing dynamic support for RTE in the context of Entity-Relationship diagrams. SelfSync is implemented in Self, a prototypebased dynamic object-oriented programming language, environment, and virtual machine. In SelfSync, the modelling and the execution take place within a unique environment. A source model and the corresponding implementation object are one and the same. Both share the same structure and the same behaviour namely its prototype and its traits, respectively. In CrossFabrik the modelling and the execution also take place within a unique environment at design as well as at running time. As SelfSync, CrossFabrik consists in a dynamic support for RTE, synchronizing a model and its implementation parts even at runtime. In CrossFabrik, the behaviour is also shared between source models and implementation classes. It is made of AST nodes that can be produced at design time and executed during the assessment of implementation classes.

Our solution differ from SelfSync in the management of the relationship between model and implementation parts. In CrossFabrik, because a source model may have several implementations, the same object cannot be used, implementa- 
tion classes have to be generated and a one-to-many relationship have to be maintained. Such a synchronization cannot be achieved with SelfSync. Moreover, SelfSync do not support a runtime RTE in the reverse direction [21]. In CrossFabrik, we provide such a reverse support especially during the debugging process. From an instance of an implementation class, the source model can be inspected and updated. Such updates affect automatically the source model, its implementation classes and their instances currently in use.

\section{Conclusion And Future Works}

This paper addresses the issue of software assessment during development in the context of Feature-Oriented ModelDriven Development. Typically, assessing a software in the context of FOMDD involves generating the final software from models and executing it separately from models.

In this paper, we presented CrossFabrik, an environment that allows debugging as well as round-trip engineering for Feature-Oriented Model-Driven Development. The CrossFabrik RTE manages a one-to-many relationship between the source models and the implementation parts, even at runtime.

We implemented our approach using the Pharo environment that provides us an image-based virtual machine in which our modelling tools and the simulation process take place. Regarding our RTE implementation, changes propagation is fully supported in the forward direction as well as the reverse direction and includes structural and behavioural changes. Such changes can be achieved statically or dynamically during assessment. Regarding the reuse of the Pharo infrastructure, we presented how existing development tools are reused and extended to allow custom visualization and dynamic debugging of source models during execution of implementation parts.

CrossFabrik is still under development. The inheritance change and the renaming of an implementation class are not supported by the reverse engineering. The future works consists in implementing these features.

\section{References}

[1] S. Trujillo, D. Batory, and O. Diaz, "Feature Oriented Model Driven Development: A Case Study for Portlets," Software Engineering, 2007. ICSE 2007. 29th International Conference on, pp. 44-53, 2007.

[2] S. Apel and C. Kästner, "An overview of feature-oriented software development," Journal of Object Technology, vol. 8, no. 5, pp. 49-84, 2009.

[3] M. Eisenstadt, "My Hairiest Bug War Stories," Commun. ACM, vol. 40, no. 4, pp. 30-37, 1997.

[4] M. Antkiewicz and K. Czarnecki, "Framework-specific modeling languages with round-trip engineering," Model Driven Engineering Languages and Systems, pp. 692-706, 2006.
[5] K. Pohl, G. Böckle, and F. Van Der Linden, Software product line engineering: Foundations, principles, and techniques, vol. 49. 2005.

[6] K. C. Kang, S. G. Cohen, J. a. Hess, W. E. Novak, and a. S. Peterson, "Feature-Oriented Domain Analysis (FODA) Feasibility Study," Distribution, vol. 17, no. November, p. 161, 1990.

[7] K. Czarnecki and U. W. Eisenecker, Generative Programming: Methods, Tools, and Applications. Addison-Wesley, 2000.

[8] J. Bosch, Design and Use of Software Architectures: Adopting and Evolving a Product-line Approach. New York, NY, USA: ACM Press/Addison-Wesley Publishing Co., 2000.

[9] Object Management Group, "OMG Meta Object Facility (MOF) Core Specification Version 2.4.1,” 2006.

[10] A. Black, S. Ducasse, O. Nierstrasz, D. Pollet, D. Cassou, and M. Denker, Pharo by Example. Square Bracket Associates, 2009.

[11] T. Verwaest, "Flexible Object Layouts Enabling Lightweight Language Extensions by Intercepting Slot Access," in $O O P$ SLA, pp. 1-14, 2011.

[12] M. M. Peck, N. Bouraqadi, S. Ducasse, L. Fabresse, and M. Denker, "Ghost: A Uniform and General-Purpose Proxy Implementation," 2013.

[13] A. Chiş, O. Nierstrasz, and T. Gîrba, "Towards a moldable debugger," Proceedings of the 7th Workshop on Dynamic Languages and Applications - DYLA '13, pp. 1-6, 2013.

[14] D. Steinberg, F. Budinsky, E. Merks, and M. Paternostro, EMF: eclipse modeling framework. Addison-Wesley Professional, 2nd ed., 2008.

[15] M. L. Crane and J. Dingel, “Towards a UML Virtual Machine: Implementing an Interpreter for UML 2 Actions and Activities," in Proceedings of the 2008 conference of the center for advanced studies on collaborative research meeting of minds - CASCON '08, p. 96, 2008.

[16] T. Mayerhofer, P. Langer, and G. Kappel, “A runtime model for fUML,” in Mrt@ Runtime, pp. 53-58, 2012.

[17] Object Management Group, "Action Language for Foundational UML (ALF), Version 1.0.1,” no. October, 2013.

[18] E. Van Paesschen, W. De Meuter, and M. D’Hondt, "SelfSync: A Dynamic Round-Trip Engineering Environment," Model Driven Engineering Languages and Systems, vol. 3713, pp. 633-647, 2005.

[19] P. Ebraert and E. V. Paesschen, "Change-Oriented Round-Trip Engineering," no. Mdd, pp. 1-6.

[20] P. Ebraert, Q. Soetens, and D. Janssens, "Change-based FODA diagrams: bridging the gap between feature-oriented design and implementation," ... of the 2011 ACM Symposium on ..., 2011.

[21] E. Van Paesschen and M. D'Hondt, "SelfSync: A dynamic round-trip engineering environment," Satellite Events at the Models 2005 Conference, vol. 3844, pp. 347-352, 2006.

[22] M. Volter, "From Programming to Modeling-and Back Again,” Software, IEEE, vol. 28, no. 6, pp. 20-25, 2011. 